\title{
Observations of Bn and An stars: New Be stars
}

\author{
K.K. Ghosh ${ }^{1,2, \star}$ K.M.V. Apparao ${ }^{3}$ and S. Pukalenthi ${ }^{1}$ \\ ${ }^{1}$ Indian Institute of Astrophysics, Vainu Bappu Observatory, Kavalur, Alangayam, Vellore, TN 635 701, India \\ ${ }^{2}$ Space Sciences Laboratory, NASA/Marshall Space Flight Center, Mail Code ES84, Huntsville, AL 35812, U.S.A. \\ ${ }^{3}$ Department of Physics, University of Bombay, Bombay 400 098, India \\ e-mail: kajal.ghosh@msfc.nasa.gov and aprao@tifrvax.tifr.res.in
}

Received August 10; accepted August 24, 1998

\begin{abstract}
From a survey of spectra of Bn/An stars, we have detected seven new Be stars: HR 1056 (A0Vn), 1544 (A1Vn), 2191 (A0Vnn), 2300 (B8Vn), 3134 (B9.5 Vn), 3878 (B0.5 IIIn) and 4552 (B9IIIpSi). $\mathrm{H}_{\alpha}$ profiles of these stars are presented. Measured values of equivalent widths, full widths at half intensity maximum, and the peakseparations of the $\mathrm{H}_{\alpha}$ emission profiles are also tabulated in this paper. We have also computed the radii of emission disks of the newly detected Be stars.
\end{abstract}

Key words: stars: emission-line, Be

\section{Introduction}

Several B and A stars in the Bright Star Catalogue (Hoffleit \& Jaschek 1982) have been designated as Bn and An stars. The " $n$ " tag was given by Adams \& Joy (1923) meaning the spectroscopic lines are "nebulous" in contrast to "sharp" lines seen in other stars. The "nebulous" nature of the lines presumably results from the fast rotation of the stars, that leads to the large broadening of the lines. Several surveys have been made to search for newly Be stars using different criteria (Irvine 1975; Irvine \& Irvine 1979; Irvine 1990; Cote \& van Kerwijk 1993 and references therein). It may be seen from both the Bright Star (Hoffleit \& Jaschek 1982) and the Be star (Jaschek \& Egret 1982) Catalogues that there are many $\mathrm{Bn}$ and An stars that have been identified as Be stars. We suspect that the Bn An stars that are listed in the Bright StarCatalogue (Hoffleit \& Jaschek 1982), may contain many latent $\mathrm{Be}$ and Be-shell stars. During January - February 1997, we have obtained high resolution $\left(\sim 0.17 \AA\right.$ pixel $\left.^{-1}\right)$ and moderate-resolution

Send offprint requests to: K.K. Ghosh

* NAS/NRC Research Associate. On leave from Indian Institute of Astrophysics. $\left(\sim 1 \AA\right.$ pixel $\left.^{-1}\right)$ spectra of $33 \mathrm{Bn}$ and 49 An stars. The selection of these Bn and An stars was dictated by the accessibility to our telescope and the limit of the spectrograph to obtain good signal-to-noise ratio spectra. Also to compare the spectra of $\mathrm{Bn}$ and An stars we obtained the spectra of $17 \mathrm{~B}$ and A stars of high rotational velocities $\left(v \sin i>200 \mathrm{~km} \mathrm{~s}^{-1}\right)$ and another $7 \mathrm{~B}$ stars with very low rotational velocities $\left(v \sin i<35 \mathrm{~km} \mathrm{~s}^{-1}\right)$, using the same instrumentation that were used for Bn and An stars. Close inspection of the spectra of $82 \mathrm{Bn}$ and An stars (Table 1 presents the list of observed $\mathrm{Bn}$ and An stars with HR and HD numbers, spectral types, $V_{\text {mag }}, B-V$ and $v \sin i$ values) reveals seven new Be stars (Be star designation also includes Oe and Ae stars). Results of spectral analysis of these seven new Be stars are presented here. Section 2 describes the observations and data analysis. Results and discussion are given in Sect. 3. Section 4 presents the conclusion.

\section{Observations and data analysis}

Spectra of the newly detected Be stars were obtained on four nights during January - February 1997, using the Universal Astronomical Grating Spectrograph (UAGS) at the Cassegrain focus and also using the Echelle spectrograph at the Coudé focus of the $1 \mathrm{~m}$ reflector of VBO, with a CCD system. Iron-Argon or Iron-Neon and Thorium-Argon source spectra were used for wavelength calibration for UAGS and Echelle spectrograph, respectively. Dome flat-field frames were obtained to remove the pixel to pixel quantum efficiency variations. The reciprocal dispersions of UAGS and Echelle spectrograph are $19.6 \AA \mathrm{mm}^{-1}$ at $\mathrm{H}_{\alpha}$ $\left(0.45 \AA\right.$ pixel $\left.^{-1}\right)$ and $7.4 \AA \mathrm{mm}^{-1}$ at $\mathrm{H}_{\alpha}\left(0.14 \AA_{\text {pixel }}^{-1}\right)$, respectively. The spectra were analyzed using IRAF software package installed on the SUN SPARC CLASSIC 
Workstation. The following steps were followed for the reductions:

i) Trimming of the bias, the flat-field and the star spectrum CCD frames.

ii) Bias subtraction from the flat-field and the star spectrum CCD frames.

iii) Normalization of the flat-field frame and the division of the star spectrum frame by the normalized flat-field frame.

iv) Extraction of different orders of star and the comparison spectra.

v) Wavelength calibration of the stellar spectra using the coefficients of a high order polynomial fit to the comparison spectra.

vi) Normalization of the stellar spectra.

vii) Measurements of different parameters (equivalent width, full width at half intensity maximum by fitting a Gaussian profile, intensities of emission and absorption components, peak-separations, etc.) of stellar absorption and emission profiles.

The error in wavelength calibration for Coudé and Cassegrain spectra was around $0.05 \AA$ to $0.10 \AA$ respectively. To reduce the error in normalization we normalized each spectrum, at least, three to four times and finally these spectra were averaged. Around $2 \%$ of the local continuum is estimated to be the error in normalization. The instrumental broadening was computed by measuring the $F W H M$ of the narrowest line of the comparison spectral lines and this value of the $F W H M$ was taken as the instrumental broadening of the spectrograph. The values of instrumental broadening for the UAGS and the Echelle spectrographs are $0.5 \AA$ and $0.2 \AA$ respectively, at $\mathrm{H}_{\alpha}$. These values were used to correct for instrumental broadening of the measured $F W H M$ values of Be stars. Using the expression of $\sigma(\mathrm{EW})$, given in Ghosh (1988) and Ghosh et al. (1990), the errors in equivalent width measurements were computed.

\section{Results and discussion}

Spectra of $33 \mathrm{Bn}, 49 \mathrm{An}$ and $24 \mathrm{~B}$ and A stars were analyzed. It has been found from the spectra of $82 \mathrm{Bn}$ and An stars that seven stars displayed the presence of emission in their $\mathrm{H}_{\alpha}$. These are the new emission-line Bn and An stars. Also we have independently detected $\mathrm{H}_{\alpha}$-emission in two more Bn and An stars (Fig. 1) that were earlier reported as emission-line stars (HR 1037, Cote \& van Kerwijk 1993 and HR 2244, Irvine 1990). Comparison of Fig. 3 of Cote \& van Kerwijk (1993) and Fig. 1 of the present paper, clearly shows that the $\mathrm{H}_{\alpha}$-emission strength of $\mathrm{HR}$ 1037 decreased between August 1990 and January 1997. However, the $\mathrm{H}_{\alpha}$-emission strength of HR 2244 increased considerably since Irvine's detection (from the comparison of Fig. 1 of the present paper and Fig. 3 of Irvine 1993).

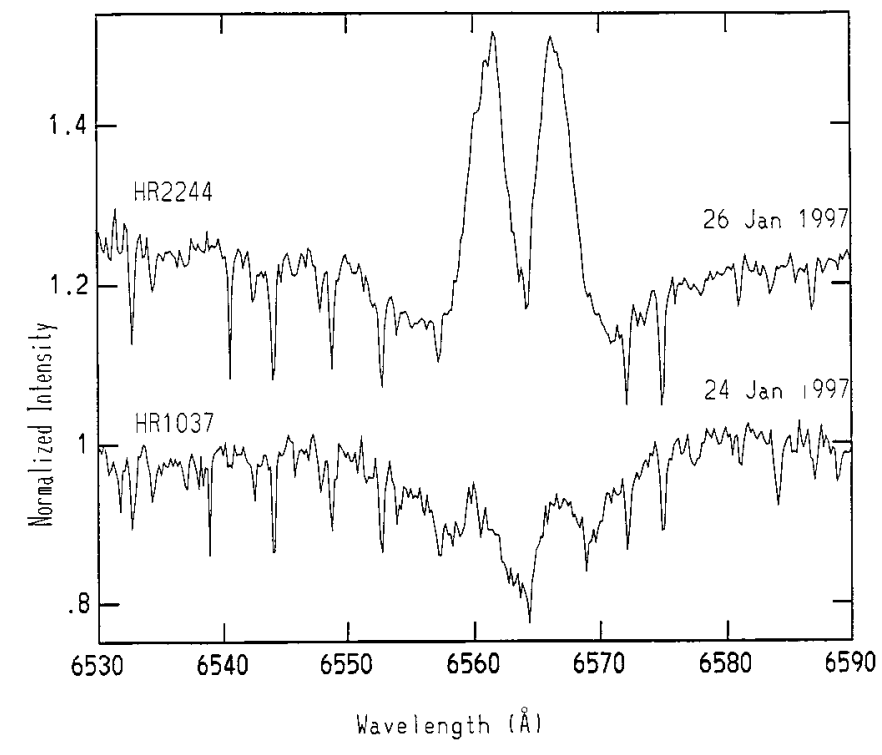

Fig. 1. Coudé Echelle $\mathrm{H}_{\alpha}$ profiles of two known Be stars, HR 1037 and HR 2244. Sharp lines present throughout the spectra are $\mathrm{H}_{2} \mathrm{O}$ telluric absorption features

Observed $\mathrm{H}_{\alpha}$-emission profiles of seven new Be stars (four Bne and three Ane stars) are shown in Figs. 2a-c. Figure 2a shows the $\mathrm{H}_{\alpha}$ emission profiles of $\mathrm{HR} 1544$ and HR 4552 that were observed using the Coudé Echelle spectrograph. It appears from this figure that the emission in $\mathrm{H}_{\alpha}$ profile may be doubtful. To check this we also obtained the spectrum of HR 2155 that has similar spectral type, luminosity class and rotational velocity as that of HR 1544. Lower part of Fig. 2b presents superimposed $\mathrm{H}_{\alpha}$ profiles of HR 1544 (A1Vn and $v \sin i=212 \mathrm{~km} \mathrm{~s}^{-1}$ ) and HR $2155\left(\mathrm{~A} 1 \mathrm{Vn}\right.$ and $\left.v \sin i=211 \mathrm{~km} \mathrm{~s}^{-1}\right)$. From the comparison of these two profiles, it may be seen that HR 1544 is an emission-line star (subtracted spectrum is presented in the upper part of Fig. 2b). Cassegrain spectra of five new Be stars are shown in Fig. 2c. We have computed the projected rotational velocities $(v \sin i)$ of three Bn and An stars, using the full width at half intensity maximum $(F W H M)$ values of He I lines $(5876 \AA$ and $6678 \AA$ ) where the He I lines have been assumed to be Gaussian profiles. Under this assumption the $v \sin i$ values were computed using the following formula (Buscombe 1969):

$$
\frac{v \sin i}{c}=\frac{F W H M}{2 \lambda_{0}(\ln 2)^{1 / 2}}
$$

where $\lambda_{0}$ is the laboratory wavelength and $c$ is the velocity of light. The $v \sin i$ values of other four Bn and An stars were obtained from the Bright Star Catalogue (Hoffleit \& Jaschek 1982). Measured equivalent widths $[W(\alpha)]$, FWHM (instrumental broadening corrected) and the peak separations $\left(\Delta V_{\text {peak }}\right)$ of seven new Be stars are given in Table 2, along with the $v \sin i$ values. We have also 

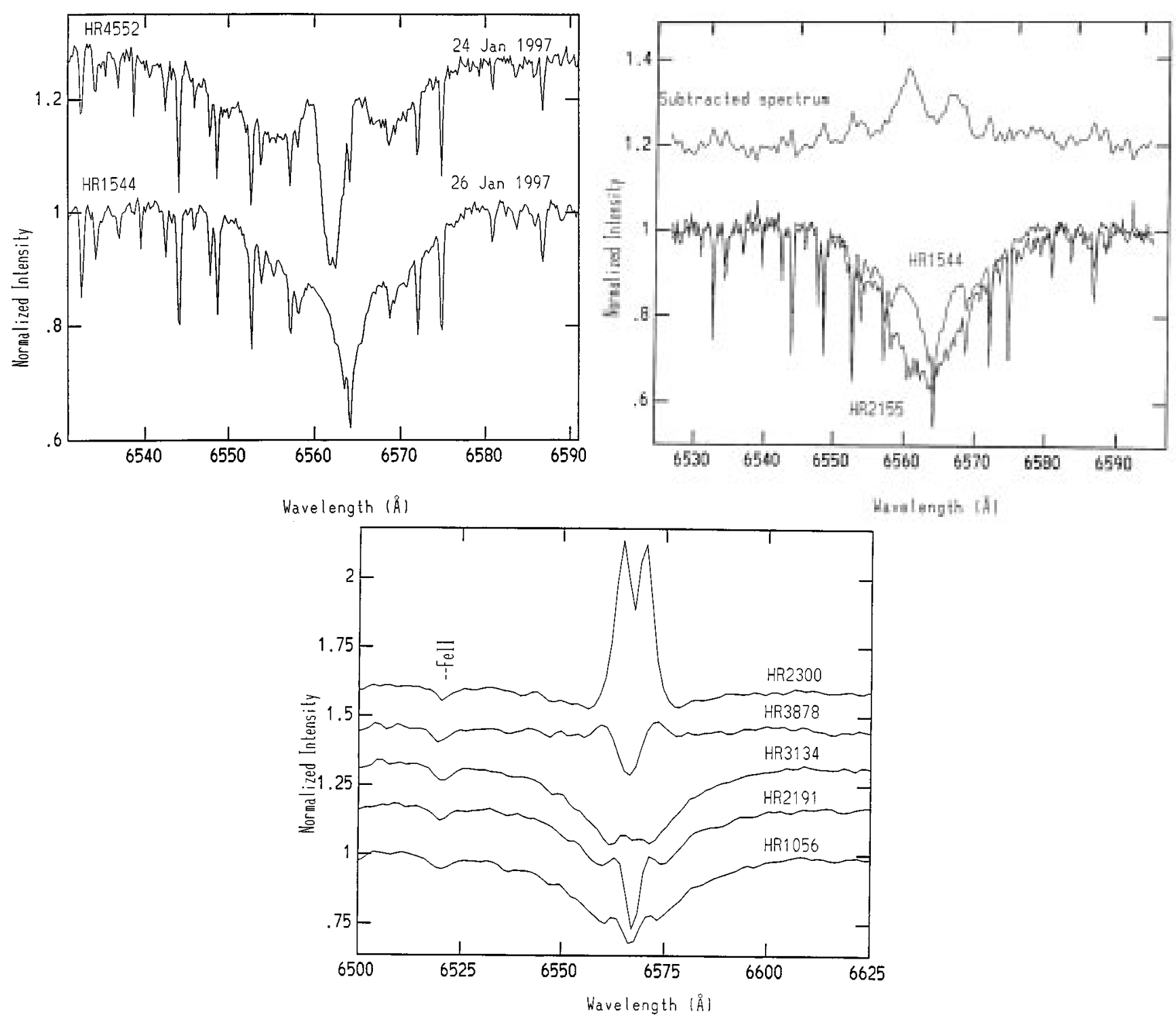

Fig. 2. a) Same as Fig. 1, but for two new Be stars, HR 1544 and HR 4552. b) Same as Fig. 1, but for the new Be star, HR 1544 and the B type star HR 2155 of similar spectral type and rotational velocity as that of HR 1544. For comparison, the $\mathrm{H}_{\alpha}$ profiles of these two stars are plotted one over the other and the subtracted spectrum (the $\mathrm{H}_{\alpha}$ profile of HR 1544 minus the $\mathrm{H}_{\alpha}$ profile HR 2155) is presented on the top that shows the presence of emission in the $\mathrm{H}_{\alpha}$ profile of HR 1544. c) Same as Fig. 1, but for the Cassegrain spectra of five new Be stars, HR 1056, HR 2191, HR 2300, HR 3134, and HR 3878

computed the radii of the emission disk of the Be stars, using the following equation (Huang 1972):

$\frac{R_{\mathrm{e}}}{R_{*}}=\left(\frac{2 v \sin i}{\Delta V_{\text {peak }}}\right)^{1 / j}$

where $R_{\mathrm{e}}$ is the radius of the emission-disk and $R_{*}$ is the photospheric radius and $j$ is an exponent that characterizes the rotation law $(j=0.5$ for Keplerian rotation of the disk and $j=1.0$ for rotation with conservation of angular momentum). To compute the values of $R_{\mathrm{e}} / R_{*}$, we have used $j=0.5$. Computed values of $R_{\mathrm{e}} / R_{*}$ of the new Be stars are given in the last column of Table 2. Errors in $W(\alpha)$ measurements were computed using the expression of $\sigma[W(\alpha)]$ given in Ghosh (1988) and Ghosh et al. (1990) and the errors in $F W H M, \Delta V_{\text {peak }}, R_{\mathrm{e}} / R_{*}$ were computed using the errors of wavelength calibration. Brief discussion about the seven new Be stars is given below:

\section{HR 1056, HD 21620 (A0Vn)}

This star has a moderate $v \sin i$ value $\left(230 \mathrm{~km} \mathrm{~s}^{-1}\right)$. The spectrum shows double-peaked weak emission inside the photospheric absorption core of the $\mathrm{H}_{\alpha}$ profile. After the photospheric absorption correction, the equivalent width of the emission component is $1.88 \pm 0.05 \AA$. Value of FWHM of the emission component, measured through 
Table 1. List of observed An/Bn stars

\begin{tabular}{|c|c|c|c|c|c|}
\hline $\mathrm{HR}$ & HD & $\begin{array}{l}\text { Spectral } \\
\text { type }\end{array}$ & $V_{\mathrm{mag}}$ & $B-V$ & $\begin{array}{l}v \sin i \\
\left(\mathrm{~km} \mathrm{~s}^{-1}\right)\end{array}$ \\
\hline 1 & 3 & $A 1 \nabla n$ & 6.71 & +0.07 & 210 \\
\hline 10 & 256 & $\mathrm{~A} 6 \nabla \mathrm{n}$ & 6.19 & +0.13 & 220 \\
\hline 26 & 560 & B9 $\sqrt{n}$ & 5.54 & -0.06 & 275 \\
\hline 55 & 1141 & B8 Vnn & 6.35 & -0.07 & \\
\hline 113 & 2626 & B9 1IIn & 5.94 & +0.01 & 225 \\
\hline 118 & 2696 & A5 Vn & 5.18 & $\frac{0.12}{-0.12}$ & 150 \\
\hline 123 & 2772 & B8 Vn & 4.73 & -0.10 & \\
\hline 266 & 5408 & B9 IVn & 5.57 & -0.09 & 178 \\
\hline 283 & 5789 & B9.5 Vn & 5.70 & -0.33 & 300 \\
\hline 336 & 6829 & A0 $V \mathrm{nn}$ & 5.29 & -0.02 & 315 \\
\hline 474 & 10161 & B9 Vn & 6.69 & -0.08 & \\
\hline 491 & 10425 & B8 IIIn & 5.78 & +0.02 & 130 \\
\hline 567 & 11946 & A0Vn & 5.26 & +0.01 & 290 \\
\hline 615 & 12885 & B9 $1 \nabla-\nabla n$ & 6.15 & -0.03 & 300 \\
\hline 708 & 15130 & B9.5 Vn & 4.89 & -0.03 & 230 \\
\hline 809 & 17036 & B9 Vn & 5.77 & -0.01 & $\frac{200}{350}$ \\
\hline 875 & 18331 & AI Vn & 5.17 & +0.08 & 300 \\
\hline 893 & 18546 & A0 $\vee \mathrm{n}$ & 6.41 & -0.03 & \\
\hline 932 & 19275 & A2 Vnn & 4.87 & +0.02 & \\
\hline 933 & 19279 & A3 Vnn & 6.41 & +0.12 & 285 \\
\hline 1037 & 21362 & $\mathrm{~B} 6 \sqrt{\mathrm{n}}$ & 5.57 & -0.03 & 385 \\
\hline 1038 & 21364 & B9 Vn & 3.73 & -0.08 & \\
\hline 1055 & 21610 & A0 $V \mathrm{n}$ & 6.57 & +0.03 & 190 \\
\hline 1056 & 21620 & A0 $\vee \mathrm{n}$ & 6.28 & +0.08 & 230 \\
\hline 1305 & 26670 & B5 $\vee \mathrm{n}$ & 5.70 & -0.14 & \\
\hline 1315 & 26793 & B9 Vn & 5.22 & -0.01 & 350 \\
\hline 1392 & 28024 & A8 Vn & 4.30 & +0.24 & 225 \\
\hline 1477 & 29459 & A5 Vn & 6.22 & +0.16 & 180 \\
\hline 1479 & 29488 & A5 Vn & 4.70 & +0.14 & 154 \\
\hline 1494 & 29722 & A1 Vn & 5.29 & 0.00 & 195 \\
\hline 1544 & 30739 & A1 $V \mathrm{n}$ & 4.36 & +0.01 & 212 \\
\hline 1565 & 31209 & A1 $V \mathrm{n}$ & 6.61 & +0.04 & 250 \\
\hline 1610 & 32040 & B9 Vn & 6.66 & -0.07 & 350 \\
\hline 1621 & 32309 & $\mathrm{~B} 9.5 \vee \mathrm{n}$ & 4.90 & -0.04 & 237 \\
\hline 1678 & 33296 & A7 Vn & 6.50 & +0.21 & 290 \\
\hline 1748 & 34748 & $\mathrm{~B} 1.5 \mathrm{Vn}$ & 6.33 & -0.10 & 280 \\
\hline 1786 & 35407 & B4 IVn & 6.32 & -0.15 & 450 \\
\hline 1798 & 35532 & $\mathrm{~B} 2 \sqrt{\mathrm{n}}$ & 6.24 & -0.07 & \\
\hline 1806 & 35640 & B9.5 Vn & 6.23 & -0.06 & 280 \\
\hline 1863 & 36646 & $\mathrm{~B} 4 \sqrt{\mathrm{n}}$ & 6.46 & -0.09 & 215 \\
\hline 1989 & 38545 & A3 Vn & 5.72 & +0.04 & 175 \\
\hline 1997 & 38670 & B9 Vn & 6.07 & -0.08 & 175 \\
\hline 1998 & 38678 & A2Vann & 3.60 & +0.05 & 245 \\
\hline 2039 & 39421 & A2 $\sqrt{n}$ & 5.97 & +0.10 & 215 \\
\hline 2155 & 41695 & A0 $V \mathrm{n}$ & 4.67 & +0.06 & 250 \\
\hline 2191 & 42477 & AO Vnn & 6.04 & 0.00 & 335 \\
\hline 2198 & 42545 & B5 $\sqrt{n}$ & 4.98 & -0.17 & 310 \\
\hline 2209 & 42818 & A0 $\vee \mathrm{n}$ & 4.80 & +0.03 & 220 \\
\hline 2244 & 43445 & B9 Vn & 5.10 & -0.08 & 235 \\
\hline 2300 & 44783 & B8 Vn & 6.26 & +0.08 & 300 \\
\hline 2324 & 45230 & A3 Vn & 5.87 & +0.08 & 250 \\
\hline 2398 & 46553 & A0 Vnn & 5.27 & -0.03 & 149 \\
\hline 2441 & 47431 & B8 IIIn & 6.57 & -0.07 & \\
\hline 2521 & 49643 & B8 IIIn & 5.75 & -0.10 & \\
\hline 2585 & 50973 & A2 Vn & 4.90 & +0.03 & 215 \\
\hline 2589 & 51104 & B8 Vn & 5.92 & -0.08 & \\
\hline 2645 & 52860 & B9 111n & 6.39 & -0.05 & \\
\hline 2751 & 56169 & A4 IIIn & 5.05 & +0.13 & 222 \\
\hline 2753 & 56221 & A5 Vn & 5.87 & +0.17 & 195 \\
\hline 2757 & 56386 & A0 $V \mathrm{n}$ & 6.19 & +0.02 & \\
\hline 2886 & 60107 & A1 $V \mathrm{n}$ & 5.25 & +0.05 & 163 \\
\hline 2901 & 60357 & AO Vnn & 5.81 & -0.02 & 240 \\
\hline 2946 & 61497 & A3 IVn & 4.99 & +0.08 & 210 \\
\hline 3008 & 62832 & A1 Vnn & 5.30 & +0.01 & \\
\hline 3039 & 63586 & AO $V \mathrm{n}$ & 6.38 & -0.18 & 325 \\
\hline 3134 & 65873 & B9.5 Vn & 5.99 & $\begin{array}{l}0.10 \\
-0.02\end{array}$ & $\frac{020}{185}$ \\
\hline 3492 & 75137 & A0 $V \mathrm{n}$ & 4.36 & -0.04 & 142 \\
\hline 3594 & 77327 & A1 Vn & 3.60 & 0.00 & 185 \\
\hline 3638 & 78702 & $\mathrm{~A} 0 / \mathrm{A} 1 \mathrm{Vn}$ & 5.73 & 0.00 & 205 \\
\hline 3766 & 82165 & A7 Vn & 6.18 & +0.23 & \\
\hline 3846 & 83650 & A0 $V \mathrm{n}$ & 6.31 & -0.03 & \\
\hline 3878 & 84567 & B0.5 IIIn & 6.45 & -0.13 & 256 \\
\hline 3889 & 85040 & A7 IVn & 6.09 & +0.25 & 18 \\
\hline 4000 & 88372 & A2 Vn & 6.25 & +0.01 & 215 \\
\hline 4024 & 88960 & A0 $V \mathrm{n}$ & 5.51 & +0.01 & 235 \\
\hline 4172 & 92245 & A0 $V \mathrm{n}$ & 6.04 & $\frac{0.00}{0.00}$ & 235 \\
\hline 4189 & 92769 & A4 Vn & 5.51 & +0.17 & 195 \\
\hline 4192 & 92825 & A3 $V \mathrm{n}$ & 5.08 & +0.09 & 154 \\
\hline 4203 & 93152 & A1 Vn & 5.24 & -0.06 & \\
\hline 4368 & 98058 & A7 IVn & 4.50 & +0.18 & 250 \\
\hline 4372 & 98161 & A3 Vn & 6.27 & +0.09 & \\
\hline 4468 & 100889 & B9.5 Vn & 4.69 & -0.06 & 192 \\
\hline 4552 & 103192 & B9 IIIpSi & 4.28 & -0.10 & 80 \\
\hline
\end{tabular}

Table 2. The new Be stars

\begin{tabular}{|l|l|l|l|l|}
\hline $\mathrm{HR}$ & $\begin{array}{l}W(\alpha) \\
(\AA)\end{array}$ & $\begin{array}{l}F W H M \\
(\AA)\end{array}$ & $\begin{array}{l}\Delta V_{\text {peak }} \\
(\AA)\end{array}$ & $R_{\mathrm{e}} / R_{*}$ \\
\hline 1056 & $01.88 \pm 0.05$ & $10.88 \pm 0.15$ & $8.83 \pm 0.12$ & $1.20 \pm 0.10$ \\
\hline 1544 & $01.30 \pm 0.04$ & $06.22 \pm 0.11$ & $7.98 \pm 0.10$ & $1.23 \pm 0.08$ \\
\hline 2191 & $02.30 \pm 0.05$ & $10.58 \pm 0.16$ & $8.70 \pm 0.12$ & $2.07 \pm 0.16$ \\
\hline 2300 & $10.62 \pm 0.13$ & $09.56 \pm 0.14$ & $5.20 \pm 0.07$ & $3.62 \pm 0.22$ \\
\hline 3134 & $01.26 \pm 0.05$ & $07.50 \pm 0.12$ & $4.10 \pm 0.05$ & $2.57 \pm 0.19$ \\
\hline 3878 & $04.88 \pm 0.07$ & $16.00 \pm 0.18$ & $6.77 \pm 0.09$ & $2.00 \pm 0.14$ \\
\hline 4552 & $01.81 \pm 0.06$ & $07.50 \pm 0.12$ & $6.05 \pm 0.08$ & $2.23 \pm 0.17$ \\
\hline
\end{tabular}

Gaussian profile fitting, is $10.88 \pm 0.15 \AA$. The peak separation between the blue and the red emission components is $8.8 \pm 0.09 \AA\left(\sim 402 \mathrm{~km} \mathrm{~s}^{-1}\right)$. Computed value of the emission disk radius is 1.2 times the stellar radius.

\section{HR 1544, HD 30739 (A1Vn)}

In the BSC, this star has been classified as a spectroscopic binary. Due to the superposition of $\mathrm{H}_{\alpha}$ profiles of two stars of the binary system may appear as a single-peak emission at the absorption core of the line. However, in the case of HR 1544, the $\mathrm{H}_{\alpha}$ profile displays double-peak emission at the core of this line which is very similar to the $\mathrm{H}_{\alpha}$ profiles of classical Be stars. Also the observed velocity difference between the two absorption cores is about $505 \mathrm{~km} \mathrm{~s}^{-1}$ and this value is not consistent with the orbital parameters. The $\mathrm{H}_{\alpha}$ profile (Fig. 2a) shows weak emission at both the wings that may not be clearly evident. To check this, we have compared the $\mathrm{H}_{\alpha}$ profiles of $\mathrm{HR} 1544$ (A1Vn and $v \sin i=212 \mathrm{~km} \mathrm{~s}^{-1}$ ) and HR $2155(\mathrm{~A} 1 \mathrm{Vn}$ and $v \sin i=$ $211 \mathrm{~km} \mathrm{~s}^{-1}$ ) and they are shown in Fig. 2b. It can be clearly seen from the subtracted spectrum (shown on the upper part of Fig. 2b) that HR 1544 displays emission at $\mathrm{H}_{\alpha}$. Measured parameters of the $\mathrm{H}_{\alpha}$-emission profile and the computed value of the radius of the emission disk are presented in Table 2.

\section{HR 2191, HD 42477 (A0Vnn)}

The $v \sin i$ value of this star is given as $160 \mathrm{~km} \mathrm{~s}^{-1}$ in the BSC. However, we have measured this value as $355 \mathrm{~km} \mathrm{~s}^{-1}$, using Eq. (1) and the FWHM value of the He I $(6678 \AA)$ line of this star. This computed value of $v \sin i$ is in good agreement with that computed using the $F W H M$ value of He I line profile of $5876 \AA$. The observed velocity difference between the two absorption cores is about $670 \mathrm{~km} \mathrm{~s}^{-1}$. This value indicates that the weak emission is not due to the superposition of two absorption line stars. The doublepeak emission of $\mathrm{H}_{\alpha}$ separated by a strong absorption component may be due to the shell absorption. The measured values of $W(\alpha), F W H M, \Delta V_{\text {peak }}$ and the computed value of $R_{\mathrm{e}} / R_{*}$ are presented in Table 2 . 


\section{HR 2300, HD 44783 (B8Vn)}

The revised value of $v \sin i\left(300 \mathrm{~km} \mathrm{~s}^{-1}\right)$ of this star is taken from the remarks part of BSC. Also our computed value of $v \sin i$, using the measured $F W H M$ value of He I $(6678 \AA)$, is in close agreement $\left(\sim 310 \mathrm{~km} \mathrm{~s}^{-1}\right)$ with this value. Strong double-peak $\mathrm{H}_{\alpha}$-emission profile is separated by an absorption component. The radius of the emission disk is almost four times the radius of this star. Table 2 shows the measured parameters of the emission profile of $\mathrm{H}_{\alpha}$.

\section{HR 3134, HD 65873 (B9.5 Vn)}

This star is a spectroscopic binary with radial velocity of about $-12 \mathrm{~km} \mathrm{~s}^{-1}$ (BSC). The rotational velocity of this star is $185 \mathrm{~km} \mathrm{~s}^{-1}$. The double-peak emission of $\mathrm{H}_{\alpha}$ suggest that this emission is not due to the superposition of two $\mathrm{H}_{\alpha}$ absorption profiles of the binary system. Also the observed velocity difference between the two absorption cores is very high $\left(\sim 450 \mathrm{~km} \mathrm{~s}^{-1}\right)$. Even though the emission is weak, but the radius of the emission disk is almost three times the radius of the star.

\section{HR 3878, HD 84567 (B0.5 IIIn)}

The $v \sin i$ value of this star is not available in the BSC. Also the SIMBAD database does not contain any information about the $v \sin i$ value of this star. We computed the value of $v \sin i$ using the $F W H M$ value of He I ( $6678 \AA$ ) profile and the computed value is $255 \mathrm{~km} \mathrm{~s}^{-1}$ (Table 2). $\mathrm{H}_{\alpha}$ profile of HR 3878 displays two broad emission components with an central absorption that is similar to quasireversal $\mathrm{H}_{\alpha}$ profiles of classical Be stars. From Table 2 it can be seen that the $F W H M$ value of the $\mathrm{H}_{\alpha}$ profile (after photospheric correction and interpolation) is about $731 \mathrm{~km} \mathrm{~s}^{-1}(16.0 \AA)$. This indicates that probably the projected rotational velocity of this star may be larger than that we have computed ( $\left.v \sin i \sim 255 \mathrm{~km} \mathrm{~s}^{-1}\right)$. Future high resolution line profile of $\mathrm{He} \mathrm{I}(4471 \AA)$ may help to resolve this problem.

\section{HR 4552, HD 103192 (B9IIIpSi)}

HR 4552 is not a Bn/An star. We observed this star as a standard star. The $\mathrm{H}_{\alpha}$ profile of this star (Fig. 2a) shows clear double-peaked emission with strong shell absorption, embedded within the photospheric absorption line. The $v \sin i$ value of HR 4552, given in BSC, is about $72 \mathrm{~km} \mathrm{~s}^{-1}$. We have also computed the value of $v \sin i$ using the $F W H M$ value of He I line $(5876 \AA)$ and our computed value is close to this value. However, using the $F W H M$ value of the $\mathrm{H}_{\alpha}$-emission profile, the computed value of $v \sin i$ is about $206 \mathrm{~km} \mathrm{~s}^{-1}$. We have used this value of $v \sin i$ to compute the value of $R_{\mathrm{e}} / R_{*}$ and it is given in Table 2 . The BSC lists this star as a double star and the second star is separated by $0.8^{\prime \prime}$ with a magnitude difference of 0.9 .

It may be possible that the seven newly detected earlytype emission-line stars, mentioned above, might have already been detected as emission-line stars. To check this, we have searched the SIMBAD database and found that no emissions have yet been detected in these seven stars. Spectral types of these seven stars are between B0.5 and A1. However, except one star (HR 3878 - B0.5IIIn) all the six stars have spectral types of B 8 or later. These B and A-type emission-line stars may be called as Be and Ae stars. Some of these Be and Ae stars may also be Herbig Ae/Be stars. To find out whether these stars belong to Herbig Ae/Be stars group or not, we have looked for IRAS $(12 \mu, 25 \mu, 60 \mu)$ fluxes associated with these seven stars. We have found from the IRAS point source catalogue (Beichman et al. 1988) that only two stars of these seven stars, were detected with IRAS (HR $1544-$ IRAS $04478+0848$ and HR 4552 - IRAS 11503 - 3337) and this was also confirmed with the search results of the SIMBAD database. $12 \mu, 25 \mu$ and $60 \mu$ fluxes of HR 1544 and HR 4552 and another 20 known Be stars were converted into magnitudes using the IRAS definition for the zero-magnitude. [12] - [25] color versus [12] - [60] color of Be stars and HR 1544 and HR 4552 were plotted and it has been found from this plot that HR 1544 and HR 4552 were occupying the same position as that of the Be stars in this color-color diagram. Also we plotted the $(V-12 \mu)$ color excess versus temperature of HR 1544 and HR 4552 and compared with the Fig. 4 of Hillenbrand et al. (1992) and find that these two stars lie in a region where the Be stars are located and the Herbig Ae/Be stars lie much above the Be stars, in this diagram. Based on these results we suggest that probably these two stars do not belong to the Herbig Ae/Be stars group. Also, based on only the $\mathrm{H}_{\alpha}$ profiles and non-detection with IRAS, it is difficult to make any comment about the Herbig Ae/Be associationship of the rest five stars (HR 1056, HR 2191, HR 2300, HR 3134 and HR 3878). Presently, we will call these seven newly detected emission-line stars as Be stars, following the definition of Be stars (rapidly rotating $\mathrm{O}, \mathrm{B}$ and A-type stars of luminosity classes III-V with Balmer emission lines are known as Be stars; details can be seen in Underhill \& Dozan 1982).

Presence of He I line (either $5876 \AA$ or $6678 \AA$ ) was detected in all the stars that are listed in Table 2, except HR 1544. It may be interesting to find out whether any of these stars be Bp He-weak or He-strong. This can be checked from the He abundance measurements. Highresolution and high signal-to-noise ratio line profiles of He I (4471 $\AA$ ) which is a photospheric line, are required to compute the He abundance. With our present data (He I lines of $5876 \AA$ or $6678 \AA$ ) it may not be possible to draw any such conclusion. 
Close inspection of $\mathrm{H}_{\alpha}$-emission profiles of these seven stars indicates that probably three of these stars may be Be-shell stars (HR 1544, HR 2191 and HR 4552).

\section{Conclusion}

From a survey of spectra of $82 \mathrm{Bn}$ and An stars (33 Bn stars and 49 An stars) we have detected seven new $\mathrm{H}_{\alpha}$ emission-line stars. Detection of these seven new Be stars will be useful to compute the frequency distribution of Be stars among the main-sequence B and A stars of the BSC. Among these seven Be stars, two have been detected as IRAS sources (HR 1544 and HR 4552). Three of these seven stars may be possible Be-shell stars (HR 1544, HR 2191 and HR 4552).

Acknowledgements. We are grateful to the referee, Myron Smith, for valuable comments and suggestions that helped to improve this paper. Sincere thanks to K. Kuppuswamy, C. Velu and A. Muniandi for their help and assistance during the observations. KMVA thanks the Indian National Science Academy for a Fellowship. He also thanks Professor Patel of the University of Bombay for his interest in the work. Part of this work was carried out while K.K. Ghosh held a National Research Council-(NASA/Marshall Space Flight
Center) Research Associateship. This research has made use of the SIMBAD database, operated at CDS, Strasbourg, France.

\section{References}

Adams W.S., Joy A.H., 1923, ApJ 57, 149

Beichman C.A., Neugebauer G., Habing H.J., Clegg P., Chester T.J., 1988, NASA Ref. Publ. - 1190, 1B

Buscombe W., 1970, MNRAS 148, 79

Cote J., van Kerkwijk M.H., 1993, A\&A 274, 870

Ghosh K.K., 1988, A\&AS 75, 261

Ghosh K.K., Pukalenthi S., Jayakumar K., Kuppuswamy K., Muniyandi A., Sanjeevkumar T., 1990, A\&AS 82, 557

Hillenbrand L.A., Strom S.E., Vrba F.J., Keene J., 1992, ApJ 397,613

Hoffleit D., Jaschek C., 1982, The Bright Star Catalogue, Yale University Observatory, New Haven (BSC)

Huang S.S., 1972, ApJ 171, 549

Irvine N.J., 1975, PASP 87, 773

Irvine N.J., 1990, PASP 102, 669

Irvine N.J., Irvine C.E., 1979, PASP 91, 105

Underhill A., Doazan V., 1982, in B Stars with and without Emission Lines, NASA SP-456, NASA Publications, Washington, Ch. 13 